\title{
On the Influence of Haemorrhage upon the Coagulation Time of Blood in Splenectomized Rabbits.
}

\author{
By \\ SHIMPEI KANOWOKA. \\ (跑 岡普 本) \\ (From the Physiological Laboratory of Prof. Y. Satake, \\ Toholu Imperial University and the Laboratory \\ of the Sendai Railuvay Hospital.)
}

A few years ago an interesting report that bleeding is no more capable of shortening the blood coagulation time when the spleen is previously removed was presented by $\mathrm{McClintock}$ and $\mathrm{Magers}$. This work on dogs was really started from the viewpoint of B a rcroft and his co-adjutors's that in the earlier stage of haemorrhage of a certain amount the spleen, by contraction, approximately compensates for a loss of blood. From his clinical experience Steph an ${ }^{33}$ gave about ten years ago a suggestion that the $\mathrm{x}$-ray radiation on the spleen acts similarly with the bleeding rê the coagulation time of blood. The present writer was led by a keen interest to acknowledge the underlying mechanism whereby the spleen is capable of abolishing the acceleration by bleeding in the coagulation time of blood, that is, to test whether it is only simply mechanical viz. by compensating the volume of circulating blood or of a biochemical nature viz. by causing changes in the factors causing the clotting. By repeating firstly the experiment of American authors on rabbits the present writer has not been able to duplicate them, as the following data show.

The coagulation time was determined by either the Cannon and Mendenhall, modified by Hirayama $a^{4}$ or the Brodie, Russel and $\mathrm{B} \circ \mathrm{g} \mathrm{g} \mathrm{s}^{\left.{ }^{5}\right)}$ method. In conducting the former the rabbit was fastened

\footnotetext{
2) Barcroft, Harris, Orahovats and Weiss, J. of Physiol, 1925, 60, 443.

3) Stepha n, Münch. m. W., 1920, 309.

4) Hiray a m a, Tohoku J. of Exp. Med., 1925, 6, 160.

5) Brodie, Russel and Boggs, J. of Physiol, 1898, 21, 403.
} 24, 203 .

1) McClintock and Magers, Proc. of Soc. for Exp. Biol. and Med., 1926(-27), 
on the back and the blood sample was drawn from the femoral artery just below the deep femoral branch, and the bleeding made from the carotid, while the blood sample for the latter method was taken from the denervated ear vein of the non-fastened animal, but the bleeding was carried out in the same manner as in another set of rabbits. The arterial as well as the venous blood was thus examined. White rabbits weighing about two kilos were experimented on, and the total blood volume was assumed as $5.44 \%$ of the body weight according to Mee and Gasser.) One fifth or one third of it was shed in the present investigation, since such quantities were proved sufficient to see a definite acceleration in the coagulation time of blood in rabbits in the hands of the previous experimentalist in this laboratory who made use of the same material and procedures as ourselves, and the results have been compared with his.? The total blood volume in rabbits is not materially altered by removing the spleen. ${ }^{8)}$

The present paper comprises in fact (1) observations of the blood coagulation time extending for some hours before and after the simple laparotomy, conducted as the control, and (2) those before and after the splenectomy and then those before and after the bleeding.

The spleen was removed per laparotomiam without narcosis, and the bleeding was tried at least 5 days after the splenectomy and the longest time intervening between the splenectomy and bleeding was 99 days. When the bleeding was conducted earlier than 6 days after splenectomy, the rabbits succumbed on bleeding the above related amount within a short spell.

(1) The coagulation time was determined in 4 normal rabbits by means of the Cannon every half hour for 5 hours; the values were $5^{\prime}$ to $6^{\prime}, 5^{\prime}$ to $6^{\prime} 15^{\prime \prime}$ and $5^{\prime} 15^{\prime \prime}$ to $6^{\prime}$ respectively in 3 rabbits, while in the first rabbit (No. 14 o), which looked active but died in fact 21 days later, it fluctuated in wide limits (from $2{ }^{\prime} 15^{\prime \prime}$ to $6^{\prime}$ ). On other days the coagulation time was first determined in some specimens, then the abdominal cavity was opened, but the wounds were sewed up again about 20-30 minutes later, which corresponds to the time consumed in the cases of splenectomy, without trying any other manipulation. No narcosis was resorted to. 30 minutes later the blood sample was taken from the femoral artery, and then it was repeated with an interval of 30 minutes for 5 hours in all. Before the laparotomy the average coagulation time was $5^{\prime} 15^{\prime \prime}, 5^{\prime} 15^{\prime \prime}$ and $4^{\prime} 30^{\prime \prime}$ respectively, and after closing the laparotomy wound the values were $4^{\prime} 45^{\prime \prime}$ to $6^{\prime}, 4^{\prime} 45^{\prime \prime}$

6) Meek and Gasser, Am. J. of Physiol., 1918, 47, 302.
7) Kamei, Tohoku J. of Exp. Med., 1930, 15, 547.
8) Furuk awa (古)), Kyoto Igakn Zasshi, 1931, 28, 179. 
TABLE

Control Ex

(Cannon-Mendenhall-

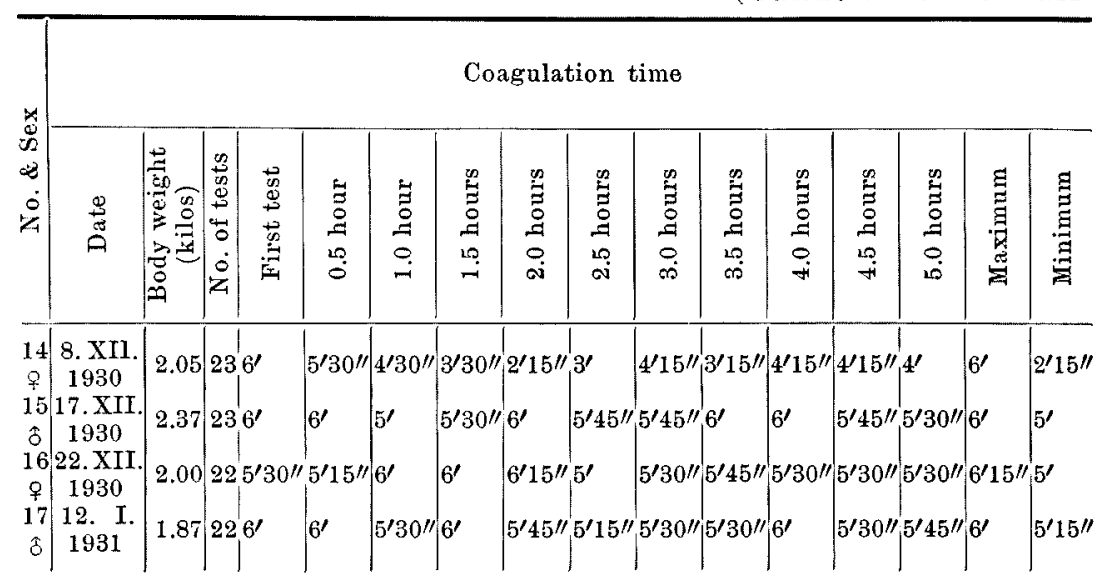

(Brodie-Russel-

\begin{tabular}{|c|c|c|c|c|c|c|c|c|c|c|c|c|c|c|}
\hline \multirow[b]{2}{*}{$\dot{z}$} & \multirow[b]{2}{*}{$\begin{array}{l}\stackrel{\star x}{D} \\
\stackrel{D}{\Omega}\end{array}$} & \multicolumn{13}{|c|}{ Coagu } \\
\hline & & Date & 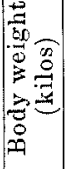 & $\begin{array}{l}0 \\
0 \\
0 \\
\Phi \\
+0 \\
0 \\
0 \\
0 \\
z\end{array}$ & 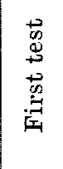 & 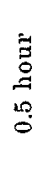 & 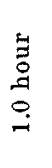 & 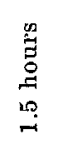 & 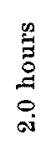 & 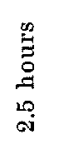 & $\begin{array}{l}\text { D. } \\
\vdots \\
\vdots \\
\vdots \\
0 \\
0 \\
\infty\end{array}$ & 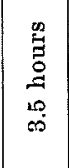 & 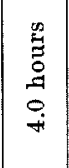 & 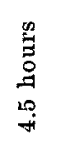 \\
\hline 28 & $\delta$ & 12. XII. 1930 & 2.05 & 23 & $2^{\prime}$ & $1 / 45 / \prime$ & $30^{\prime \prime}$ & $145 \prime \prime$ & $130 \prime$ & $145 \prime \prime$ & $2^{\prime}$ & $1^{\prime} 45^{\prime \prime}$ & $1 / 30^{\prime \prime}$ & $1^{\prime} 30^{\prime \prime}$ \\
\hline 29 & $\delta$ & 13. XII. 1930 & 2.14 & 22 & $2{ }^{\prime} 15^{\prime \prime}$ & $2 / 30^{\prime \prime}$ & $15 "$ & $2 / 15 \prime$ & $2^{\prime}$ & $2^{\prime}$ & $2 / 30^{\prime \prime}$ & 2 & $2 / 30^{\prime \prime}$ & \\
\hline 30 & $\delta$ & 19. XII. 1930 & 2.2 & 22 & $230^{\prime \prime}$ & $245^{\prime \prime}$ & & $2 / 30^{\prime \prime}$ & $2 / 30^{\prime \prime}$ & $230^{\prime \prime}$ & $20^{\prime} 30^{\prime}$ & $22^{\prime \prime}$ & 2 & \\
\hline
\end{tabular}

\begin{tabular}{|c|c|c|c|c|c|c|c|c|}
\hline \multirow[b]{2}{*}{$\dot{0}$} & \multirow[b]{2}{*}{ 芦 } & \multicolumn{4}{|c|}{$\begin{array}{l}\text { Coagulation time just before } \\
\text { simple laparotomy }\end{array}$} & \multirow[b]{2}{*}{ 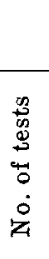 } & \multirow[b]{2}{*}{$\begin{array}{l}\ddot{\Xi} \\
\vdots \\
0 \\
0\end{array}$} & \multirow[b]{2}{*}{ 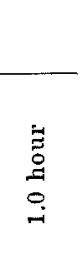 } \\
\hline & & Date & 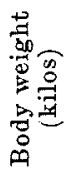 & 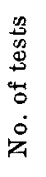 & 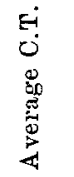 & & & \\
\hline 28 & 3 & 17. I. 1931 & 2.2 & 2 & $1 / 30^{\prime \prime}$ & 18 & $1^{\prime} 30^{\prime \prime}$ & $1^{\prime} 30^{\prime \prime}$ \\
\hline 29 & $\delta$ & 6. II. 1931 & 2.29 & 3 & $2 / 10^{\prime \prime}$ & 20 & $2^{\prime}$ & $2 / 15^{\prime \prime}$ \\
\hline 30 & $\hat{\delta}$ & 6. II. 1931 & 2.45 & 3 & $2^{\prime} 30^{\prime \prime}$ & 20 & $2 \prime 15^{\prime \prime}$ & $2^{\prime}$ \\
\hline
\end{tabular}


I.

periments.

Hirayama's method)

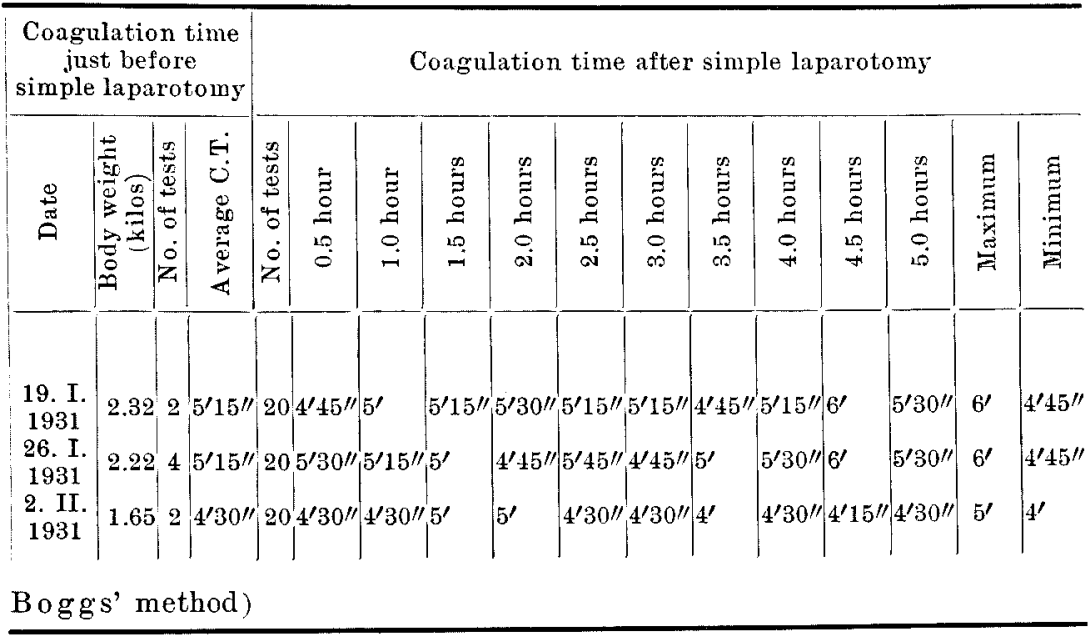

lation time

\begin{tabular}{|c|c|c|c|c|c|c|c|c|c|c|c|c|c|c|c|}
\hline 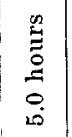 & 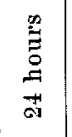 & $\begin{array}{l}\infty \\
\underset{⿱ 宀}{0} \\
\infty \\
\infty\end{array}$ & $\begin{array}{l}\text { 怘 } \\
\stackrel{5}{0} \\
0 \\
\infty\end{array}$ & 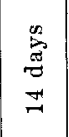 & $\begin{array}{l}\text { 点 } \\
\text { 焉 } \\
\stackrel{2}{n}\end{array}$ & 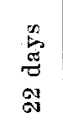 & 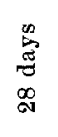 & 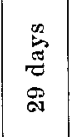 & 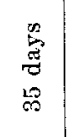 & 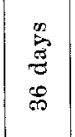 & 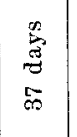 & $\begin{array}{l}\frac{0}{2} \\
\frac{6}{0} \\
0 \\
0\end{array}$ & $\begin{array}{l}\stackrel{0}{5} \\
\stackrel{5}{5} \\
0 \\
10\end{array}$ & 莺 & 䔍 \\
\hline $1^{\prime} 30^{\prime \prime}$ & $1^{\prime} 45^{\prime \prime}$ & & $\begin{array}{c}1^{\prime} 22^{\prime \prime} \\
\text { (4) }\end{array}$ & & $\mid \begin{array}{r}1^{\prime} 30^{\prime \prime} \\
\text { (4) }\end{array}$ & & & $\left|\begin{array}{r}1 \\
1 \\
(4)\end{array}\right|$ & & $\begin{array}{r}1 / 30 \prime \prime \\
\text { (4) }\end{array}$ & $\mid \begin{array}{r}130^{\prime \prime} \\
(2)\end{array}$ & & & & $1^{\prime} 22^{\prime \prime}$ \\
\hline $\begin{array}{l}2^{\prime} 15^{\prime \prime} \\
25^{\prime \prime \prime}\end{array}$ & $2^{\prime \prime}$ & {$\left[\begin{array}{c}2^{\prime} 15^{\prime \prime} \\
(4) \\
2^{\prime} 15^{\prime \prime} \\
(4)\end{array}\right.$} & & $\begin{array}{c}2^{\prime} 15^{\prime \prime} \\
\text { (4) }\end{array}$ & & $\underset{(4)}{22^{\prime 2} 2^{\prime \prime}}$ & (4) & 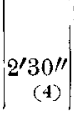 & $\quad(4)$ & & & $\left|\begin{array}{r}230 \prime \prime \\
(3)\end{array}\right|$ & $\left.\right|^{2 / 10^{\prime \prime}}$ & $\mid \begin{array}{l}2 / 30^{\prime \prime} \\
2^{\prime} 45^{\prime \prime}\end{array}$ & $2^{2}$ \\
\hline
\end{tabular}

Coagulation time after simple laparotomy

\begin{tabular}{|c|c|c|c|c|c|c|c|c|c|}
\hline 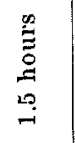 & 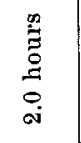 & 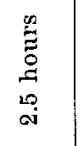 & $\begin{array}{l}\stackrel{\infty}{0} \\
\stackrel{\Xi}{\Xi} \\
\vdots \\
0 \\
\infty\end{array}$ & 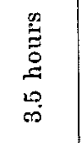 & $\begin{array}{l}\stackrel{0}{0} \\
\stackrel{\Xi}{0} \\
\stackrel{9}{9} \\
\stackrel{+}{+}\end{array}$ & 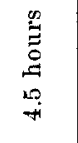 & 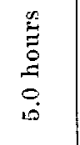 & 吉 & 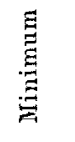 \\
\hline $1^{\prime} 30^{\prime \prime}$ & $1^{\prime} 45^{\prime \prime}$ & $1^{\prime} 15^{\prime \prime}$ & $1^{\prime} 30^{\prime \prime}$ & $1^{\prime} 45^{\prime \prime}$ & $\mathbf{1}^{\prime} 45^{\prime \prime}$ & & $1^{\prime} 30^{\prime \prime}$ & $1^{\prime} 45^{\prime \prime}$ & $1^{\prime} 15^{\prime \prime}$ \\
\hline $2^{\prime}$ & $2^{\prime} 15^{\prime \prime}$ & $20^{\prime \prime}$ & $\dot{2}$ & $1^{\prime} 45^{\prime \prime}$ & $g^{\prime} 15^{\prime \prime}$ & $2^{\prime}$ & $2^{\prime}$ & $2 / 30^{\prime \prime}$ & $1^{\prime} 45^{\prime \prime}$ \\
\hline $2^{\prime}$ & $2^{\prime}$ & $2^{\prime}$ & $2^{\prime} 15^{\prime \prime}$ & $2^{\prime} 30^{\prime \prime}$ & $2^{\prime}$ & $1^{\prime} 45^{\prime \prime}$ & $2^{\prime}$ & $2 / 30^{\prime \prime}$ & $1^{\prime} 45^{\prime \prime}$ \\
\hline
\end{tabular}




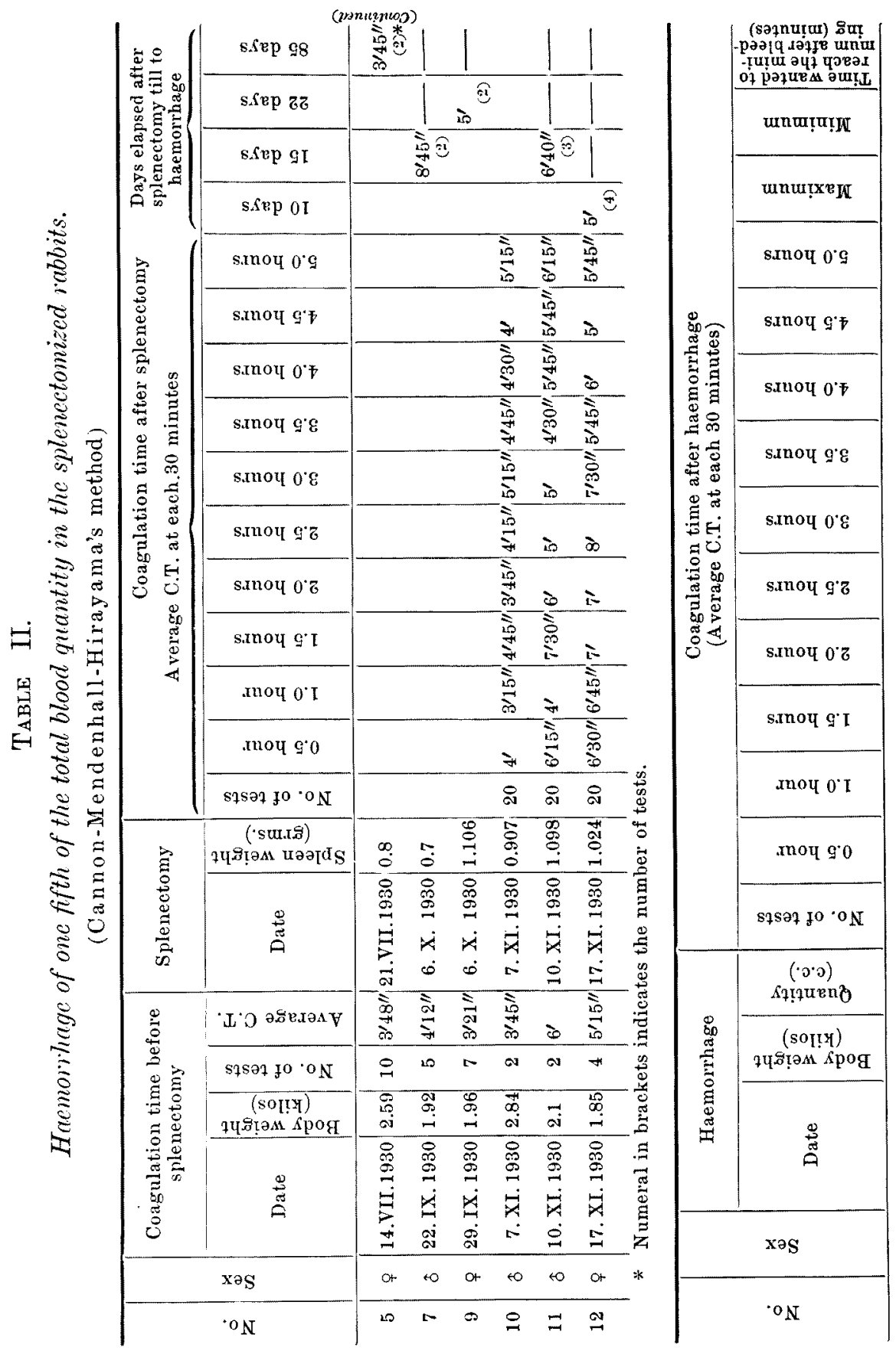




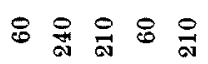

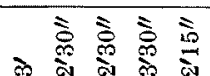

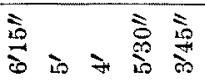

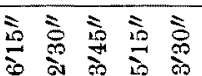

弯意离的高

的高离高高高

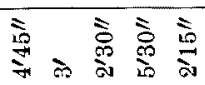

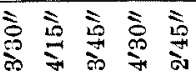

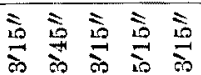

高离的的

高高言竞

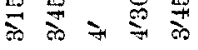

小的为离高

高的东离蹗

ㅇํㅇ요용요

ธิง

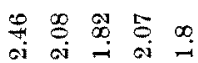

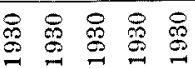

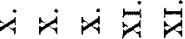

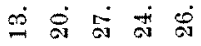

at to of to ot

$0-\sigma=0$

\begin{tabular}{|c|c|c|c|}
\hline & s.sep 66 & $=\hat{3}$ & \\
\hline 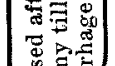 & $\operatorname{sictp} \tau_{2}$ & 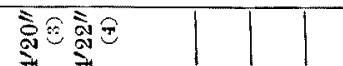 & \\
\hline & ssipp oq & 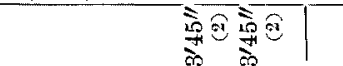 & \\
\hline 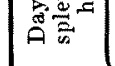 & ssep 08 & 产方 & \\
\hline & $\operatorname{sipp} 66$ & $=8$ & \\
\hline & ssep 98 & & \\
\hline & $\operatorname{sspp} 62$ & & \\
\hline & $\operatorname{sisp} t 2$ & 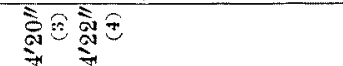 & \\
\hline & 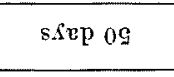 & 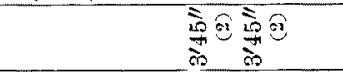 & \\
\hline 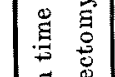 & $\operatorname{sisp} \ldots F$ & 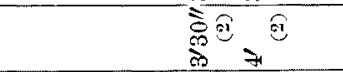 & \\
\hline 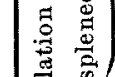 & 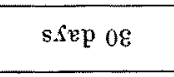 & 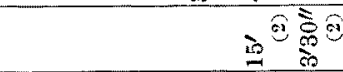 & \\
\hline 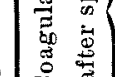 & $\operatorname{ssep}_{6 z}$ & 彎 & 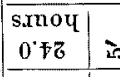 \\
\hline 罚 & ssep $\varepsilon z$ & & 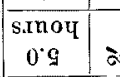 \\
\hline & ssipp az & 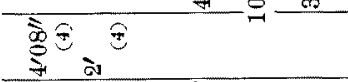 & 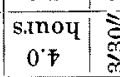 \\
\hline & sSep 91 & 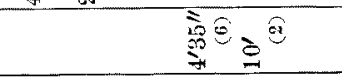 & 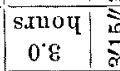 \\
\hline & s.sep $\varrho_{\mathrm{T}}$ & 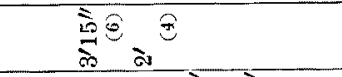 & 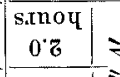 \\
\hline & ${ }_{8 \operatorname{sep} 6} 6$ & & 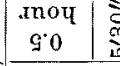 \\
\hline & s.sep 8 & 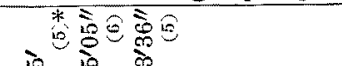 & 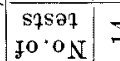 \\
\hline & 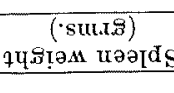 & 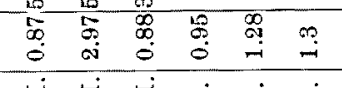 & $=$ \\
\hline 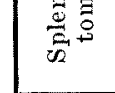 & 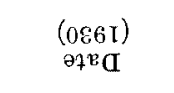 & 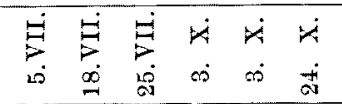 & $=$ \\
\hline 气言 & 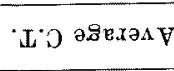 & 言言高言言 & $=$ \\
\hline 等 & 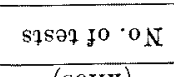 & $\Rightarrow 100+\infty$ & $=$ \\
\hline 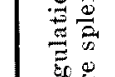 & 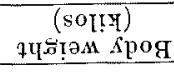 & 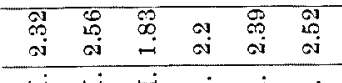 & $=$ \\
\hline 侻 & 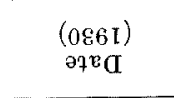 & 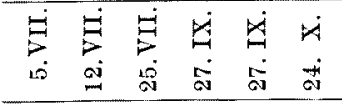 & $=$ \\
\hline & $\frac{1}{x^{\circ} \mathrm{O}}$ & 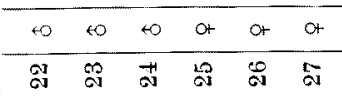 & $\begin{array}{l}= \\
=\end{array}$ \\
\hline
\end{tabular}




\begin{tabular}{|c|c|c|}
\hline & 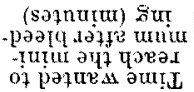 & 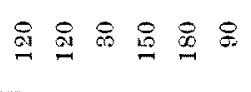 \\
\hline & unurntụtr & 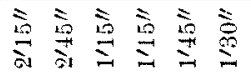 \\
\hline & umurnests & 言言言言言高 \\
\hline & s.moप $0^{\circ} \mathrm{g}$ & 今े 今े \\
\hline 㱐 & sanot $g^{\circ} \neq$ & 率商商密 \\
\hline 节. & s.Inoy $0^{\circ} T$ & 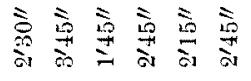 \\
\hline 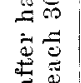 & s.moy g'E & 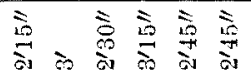 \\
\hline 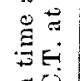 & s.mol $0^{\circ} 8$ & 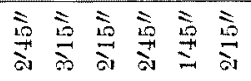 \\
\hline 焉 & sinoy 96 & 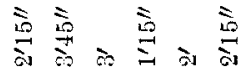 \\
\hline & s.mot $0 \%$ & 言言言言高 点 \\
\hline & S.tnoy g. I & 言言言言言商 \\
\hline & anou $0^{\circ} \mathrm{r}$ & 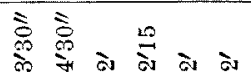 \\
\hline & snoy co & 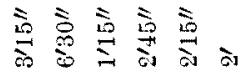 \\
\hline & s7sat go $\circ \mathrm{N}$ & $\stackrel{\circ}{\rightarrow} \cong$ 요 \\
\hline & 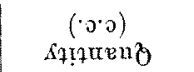 & $\stackrel{\infty}{a} \stackrel{\infty}{a} \vec{a} \stackrel{a}{a}$ \\
\hline$\stackrel{8}{3}$ & $\begin{array}{c}\text { (sor!y) } \\
74 \text { s! } \mathrm{spog}\end{array}$ & 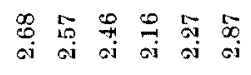 \\
\hline$\stackrel{\Xi}{\stackrel{\Xi}{9}}$ & 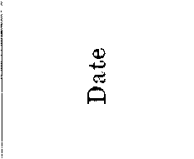 & 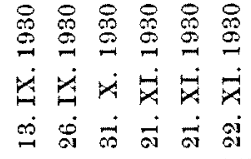 \\
\hline & xas & to to to ot ot of \\
\hline & $o_{N}$ & 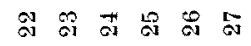 \\
\hline
\end{tabular}

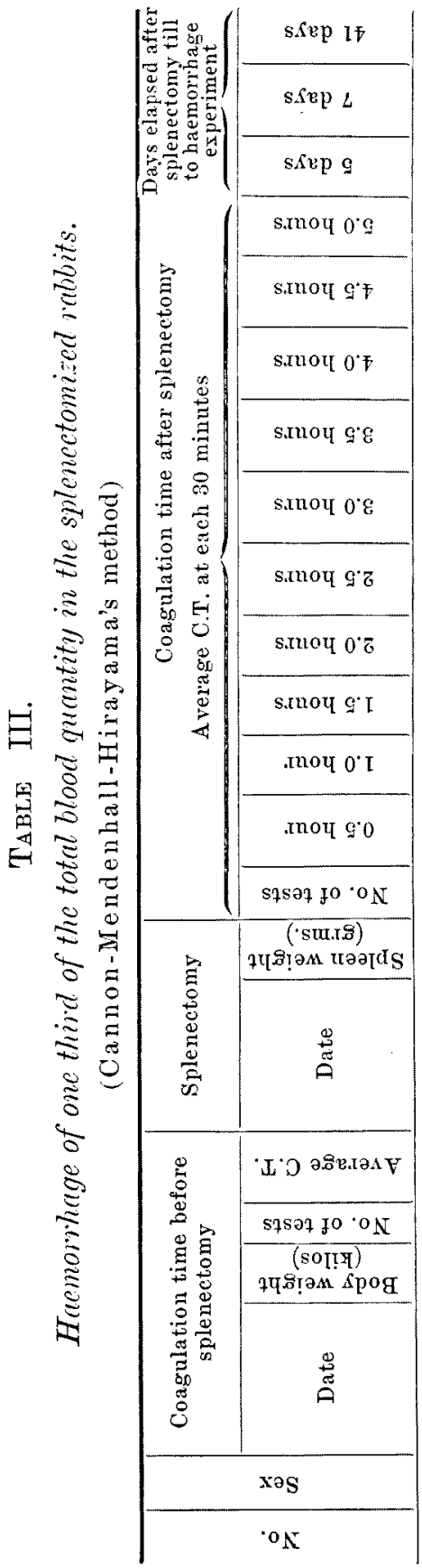




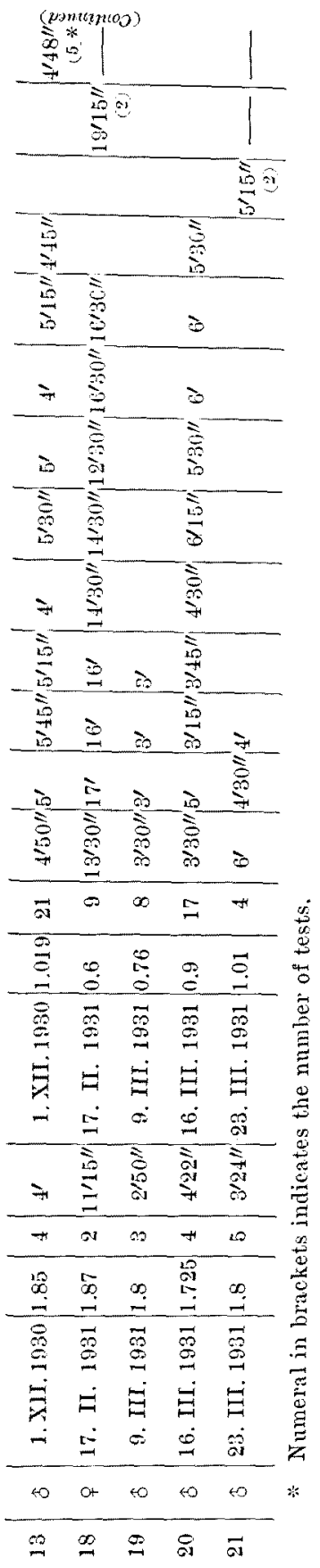

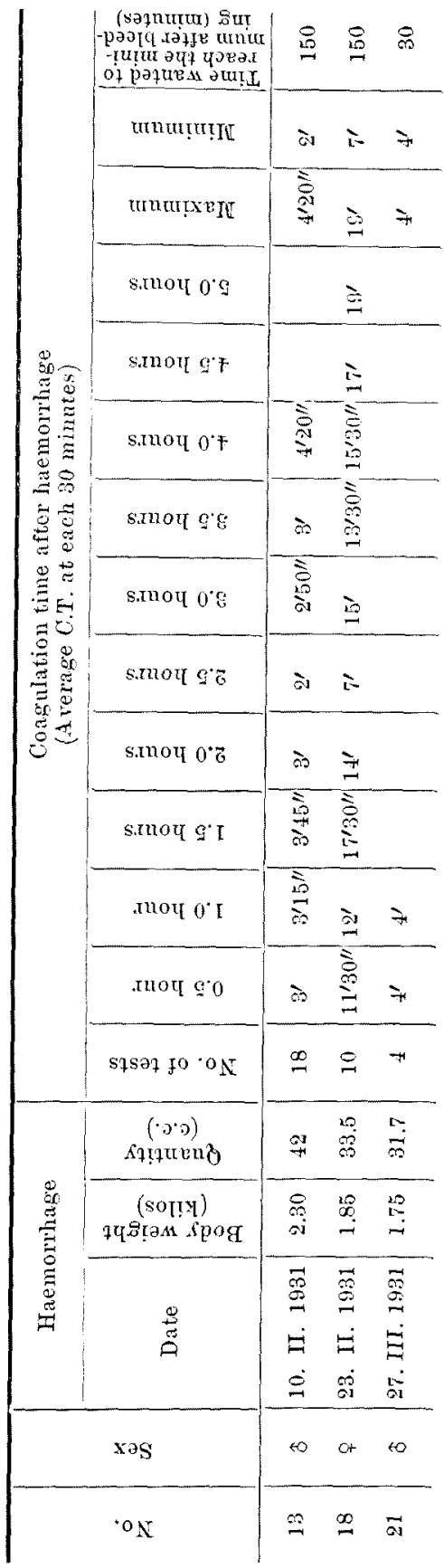




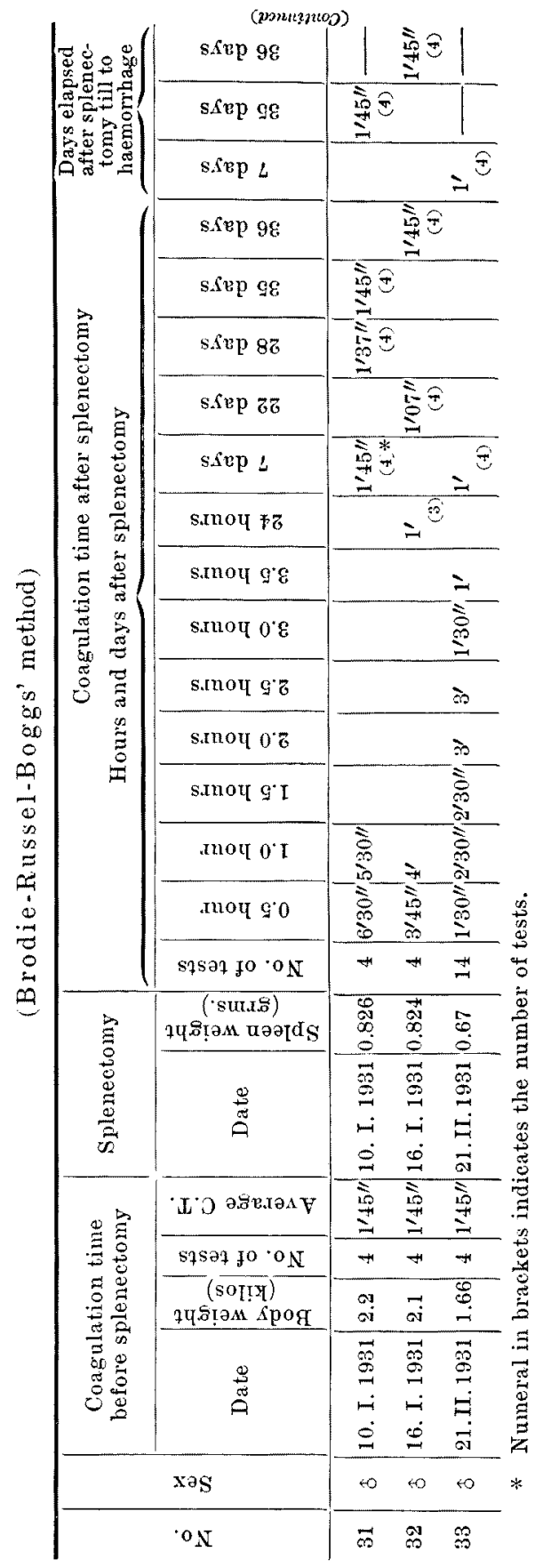

\begin{tabular}{|c|c|c|c|c|}
\hline \multirow{14}{*}{ 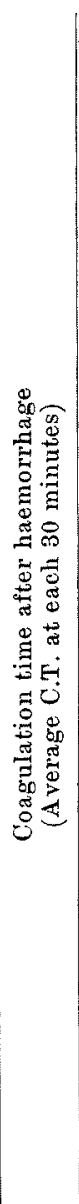 } & 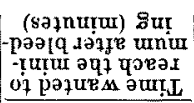 & 8 & 8 & 8 \\
\hline & umurịụ & डे & ఫे & ఫे \\
\hline & 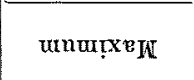 & 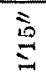 & $\Rightarrow$ & $=$ \\
\hline & s.lnoy $0^{\circ} \mathrm{C}$ & $\stackrel{\text { in }}{\stackrel{10}{\mid}}$ & है & $=$ \\
\hline & s.moप 9 & 这 & 穼 & $=$ \\
\hline & sinoy $0^{\circ} \theta$ & $=$ & $=$ & $=$ \\
\hline & s.moy 98 & 高 & $=$ & 二 \\
\hline & s.moy $0^{\circ} 8$ & $=$ & is & $=$ \\
\hline & s.moy $g^{\prime}$ & 高 & 密 & ì \\
\hline & s.tnoy $0^{\circ} z$ & îे & î̀ & $=$ \\
\hline & s.tnoप $\varrho^{\prime} I$ & 言 & 㐔 & is \\
\hline & хиоч $0^{\circ} \mathrm{I}$ & 商 & 商 & 亏े \\
\hline & mоч go & : & $=$ & $=$ \\
\hline & s7saz jo $0^{\circ} \mathrm{N}$ & \& & 8 & จे \\
\hline \multirow{3}{*}{$\equiv$} & $\begin{array}{c}(\cdot 0 \cdot 0) \\
\text { sqtąueng }\end{array}$ & 采 & $\begin{array}{l}0 \\
\dot{j} \\
\dot{\forall}\end{array}$ & $\overbrace{8}^{\infty}$ \\
\hline & 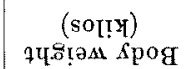 & 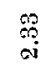 & 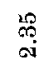 & : \\
\hline & 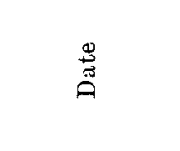 & $\begin{array}{l}\vec{g} \\
\stackrel{g}{-} \\
\vec{g} \\
\dot{g}\end{array}$ & $\begin{array}{l}\overrightarrow{8} \\
\vec{g} \\
\vec{g} \\
\dot{g}\end{array}$ & 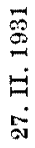 \\
\hline & xas & $\leqslant 0$ & 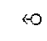 & to \\
\hline & ${ }^{\circ} \mathrm{N}$ & $\vec{s}$ & s & $\infty$ \\
\hline
\end{tabular}


to $6^{\prime}$ and $4^{\prime}$ to $5^{\prime}$ for five hour's respectively; from these numerals and investigation of the time course in the coagulation time on Table I it can be deduced that simple laparotomy does not materially alter the blood coagulation time of rabbits. The results obtainable by use of the $\mathrm{Brodie}$ ran in the same direction; moreover the coagulation time determination in normal animals was carried out with this method for a long period, as twenty to fifty days or more. The normal values for three male rabbits were from $2^{\prime}$ to $1^{\prime} 22^{\prime \prime}, 2^{\prime} 30^{\prime \prime}$ to $2^{\prime}$ and $2^{\prime} 45^{\prime \prime}$ to $2^{\prime}$ respectively, and just before opening the abdominal cavity they were $1^{\prime} 30^{\prime \prime}, 2^{\prime} 10^{\prime \prime}$ and $2^{\prime} 30^{\prime \prime}$ respectively and after closing the cavity from $1^{\prime} 45^{\prime \prime}$ to $1^{\prime} 15^{\prime \prime}, 2^{\prime} 30^{\prime \prime}$ to $1^{\prime} 45^{\prime \prime}$ and $2^{\prime} 30^{\prime \prime}$ to $1^{\prime} 45^{\prime \prime}$ respectively. No definite variation could thus be detected in the coagulation time of blood determinable by both the methods, the Cannon and the Brodie by simple laparotomy of a short duration. In passing it may be noted by way of precaution that the coagulation time estimated by any method has a value only relative to other determinations by one and the same method.

(2) Next the data before and after splenectomy, given in Tables II and III, will be discussed ; first the coagulation time was estimated before any other malmanipulation was carried out on the animal body, then the spleen was removed without narcosis and with as little loss of blood as possible, and the wound closed. About 20-30 minutes were occupied in this operation. In all the series with the method of $\mathrm{Can}$ non and in half of the cases with Brodie, 30 Minutes later the blood sample was taken, which was further followed by collecting a specimen with a 30 minutes interval for 5 hours and later just prior to the haemorrhage experiment that is 5 to 85 days after splenectomy, and in the other half of the cases of the Brodie the estimation was done several times during a course of many days as 7 to 99 days.

Of 12 cases, where the coagulation time was investigated for a short period of time as 5 hours, a prolongation of clotting was noted with definiteness in over half of the instances, Nos. 12, 27, 18, 20, 31, 32 and 33 , and in the rest the variation was too small to speak of occurrence of either acceleration or retardation. Further it may be added that in all cases, 4 in number, with the Brodie method the retardation was always discovered in the clotting time. When the estimation was carried out in 17 individuals 5 to 99 days after splenectomy a remarkably prolonged clotting was found on 15th day in No. 7 , on 9 th in No. 25, on 9.-30. days in No. 26, on the 9th in No. 27 and on the 7 th in No. 18, and a remarkably shortened on the 22nd day in No. 23 and on the 15th in No. 24.

The outcome was not so uniform, but it may be said that the blood of rabbit in the majority of cases clots somewhat slowly during some 
hours after removal of the spleen. Our results agree, roughly speaking, with those of Takasaki, Yunoki and Ohtsuka on rabbits." Shortening of clotting was observed after splenectomy in rabbits, for some hours thereafter by $\mathrm{A} \mathrm{kai^{10 }}$ and for some days by Yama moto. ${ }^{11)}$ According to Shichid ${ }^{19}$ the coagulation time of rabbits remains unaltered on removal of the spleen, but is largely prolonged on ligating the arteries to the spleen. The experimentalists here referred to determined the coagulation time of the venous blood.

We are not able at present to give any suggestion why quite different features were yielded in the hands of such investigators as $\mathrm{Ak}$ a $\mathrm{i}$ and $\mathrm{Y} \mathrm{a} \mathrm{m} \mathrm{a} \mathrm{moto}$ who made use of the same material and similar procedures for removing the spleen. The method for estimating the clotting time used by them was a modification of Wright by Akai himself or a modification of $\mathrm{Bürker}$ by Oinu$m a$, teacher of Yama moto. Is it justifiable to take the different methods as the cause of the different results? Not likely!

2 or $t$ weeks or two days after splenectomy the coagulation time of three patients was found by Wölisch lying within normal limits. In the third case in small retardation was discovered. ${ }^{13}$ )

19 days after splenectomy the coagulation time of a dog of $\mathrm{McClintock}$ and Magers was wholly the same as before. ${ }^{1}$ The dogs of Hamaguchi required an excessively long time after bleeding from a hole made in the ear vein by means of a needle before the bleeding stopped after the spleen was extirpated. ${ }^{1+1}$

(3) Lastly the coagulation time on bleeding in splenectomized rabbits will be referred to now. In 11 rabbits one fifth of the total blood quantity was shed, and in 6 one third. In the former 10-99 days and in the latter 5-41 days were allowed to elapse between splenectomy and the haemorrhage experiment. About 3-5 minutes were needed to shed the above related amount of blood. Before haemorrhage some blood specimens were tested, and afterwards the determinations were carried out with a 30 minutes interval. In No. 5 only a small shortening became manifest one hour after haemorrhage to one fifth,

9) Tak a s ki, Tokyo Igakkwai Zasshi, 1921, 35, 755 (Proc.), Mitt. med. Fak. Univers. Tokyo, 1923, 30, 331 (He noted: we were able to observe a remarkable prolongation of the clotting time of blood in a rabbit after splenectomy. The time elapsing after the operation till to the estimation was not noted, therefore whether it is proper to quote the outcome as above, is questionable), $Y$ un o $k \mathrm{i}$, Journal of Kinki Gyn. Soc., 1927, 10, 955 (Jap. with English abstract), Ohtsuka, Igaku Chuoh Zasshi, 1928, 26, 569.

10) A kai, Hokuetsu Igakkwai Zasshi, 1923, 38. year 262 (proc.), Nippon Gekwagakkwai Zasshi, 1924-25, 25. year, 358 .

11) Ya m a moto, Okayama-Igakkwai-Zasshi, 1930, 42, year, 477. (with German abstract).

12) Shichida, Fukuoka Ikwa Daigaku Zasshi, 1923, 16, $331 \mathrm{ff}$ and $33 \mathrm{ff}$ (with German abstract, p. 10).

13) Wölisch, Münch. m. W., 1921, 228 and 941.

14) H a m a g u chi, Tokjo Igakwai Zasshi, 1925, 39, 522. 
but in Nos. 7, 9, 11 and 12 the shortening was remarkable, $8^{\prime} 45^{\prime \prime}$ to $2^{\prime} 30^{\prime \prime}$ in No. 7 at the end of four hours after bleeding, $5^{\prime}$ to $2^{\prime} 30^{\prime \prime}$ in No. 93.5 hours, $6^{\prime} 40^{\prime \prime}$ to $3^{\prime} 30^{\prime \prime}$ in No. 11 one hour and $5^{\prime}$ to $2^{\prime} 15^{\prime \prime}$ in No. 123.5 hours. In the rabbits examined with the Brodie method and bled to one fifth the same held true with the above statement, that is the bleeding resulted invariably in shortening the coagulation time; No. $22: 4^{\prime} 20^{\prime \prime}$ to $2^{\prime} 15^{\prime \prime}$ at the end of two hours after bleeding, No. 23 : $4^{\prime} 22^{\prime \prime}$ to $2^{\prime} 45^{\prime \prime} 2$ hours, No. $24: 7^{\prime}$ to $1^{\prime} 15^{\prime \prime} 30$ Minutes, No. $25: 3^{\prime} 45^{\prime \prime}$ to $1^{\prime} 15^{\prime \prime} 2.5$ hours, No. $26: 3^{\prime} 45^{\prime \prime}$ to $1^{\prime} 45^{\prime \prime} 3$ hours and No. $27: 3^{\prime} 30^{\prime \prime}$ to $1^{\prime} 30^{\prime \prime} 1.5$ hours.

When one third of the total blood amount was lost, shortening was also invariably discovered and in a large degree. With the Canno method, No. $13: 4^{\prime} 48^{\prime \prime}$ to $2^{\prime}$ at the end of 2.5 hour's, No. $18: 19^{\prime} 15^{\prime \prime}$ to $7^{\prime} 2, \overline{5}$ hours and No. $21: 55^{\prime} 15^{\prime \prime}$ to $4^{\prime} 30$ minutes after bleeding (The animal died 1.5 hours after haemorrhage which was undertaken on the 5th day after splenectomy.); and with the Brodie method, No. 31: $1^{\prime} 45^{\prime \prime}$ to $30^{\prime \prime}$ at the end of one hour after bleeding, No. $32: 1^{\prime} 45^{\prime \prime}$ to $30^{\prime \prime}$ 1.5 hours and No. $33: 1^{\prime}$ to $30^{\prime \prime}$ one hour.

The degree of shortening in the coagulation time due to bleeding in these rabbits without the spleen is not only not inferior to that seen by $\mathrm{Kamei} \mathrm{i}^{\mathrm{T}}$ in rabbits, normal as well as bilaterally splanchnictomized, but apparently exceeds that in cases of bleeding of one fifth in his normal rabbits. Therefore it can be said with definiteness that splenectomy never interferes with the appearance and magnitude of shortening in the coagulation time of rabbits due to bleeding, contrary to the experiments of $\mathrm{McClintock}$ and $\mathrm{Magers}$ on dogs. ${ }^{1}$ In the present experimentation 5 days or more were allowed to elapse between splenectomy and the bleeding as also in McClintock and Mager's experiments. In them 110 c.c. blood was shed from a dog weighing 5.2 kilos on the day of splenectomy carried out 10 days before, and 235 c.c. from another of 10.8 kilos; about one fifth of the total blood was shed also. According to Meek and Gasser the total blood of dogs corresponds to one tenth of the body weight.") Before splenectomy a loss of 39 c.c. blood caused a remarkable shortening in the coagulation time in a small dog, weighing 5.2 kilos on the day of splenectomy carried out 19 days afterwards. With Saito and T a chi, Kame i ${ }^{152}$ shed, in passing it may be noted, about one third of the total blood in 6 dogs and came to witness a distinctly increased coagulability in half of the cases (Dogs, I, III \& IV).

Further it is also of interest to point out that the lengthening in the coagulation time after bleeding was observed in our splenectomi-

15) Saito, Ka mei and T a ch i, Tohoku J. of Exp. Med., 1928, 11, 205. 
zed rabbits only exceptionally, that is only in Nos. 5 and 23 , while it was rather frequent in the rabbits, normal as well as doubly splanchnectomized, of Kamei.

\section{Sumary.}

Splenectomy does not ever interfere with the shortening in the coagulation time of blood of rabbits, which commonly takes place on bleeding of a certain amount. In the present investigations the bleeding experiment was carried out 7 days or later after splenectomy, and one fifth or one third of the total blood volume was shed.

The lengthening in the coagulation time beyond the initial, seen after bleeding in a spell of 5 hours, almost invariably disappears after splenectomy.

A few hours after splenectomy the coagulation time was observed somewhat delayed in over half the instances. 